\title{
Transfer, Segregation and Deposition of Heavy Particles in Horizontal and Vertical Turbulent Channel Flows
}

\author{
Cristian Marchioli, Alfredo Soldati \\ Dipartimento di Energetica e Macchine \\ University of Udine, 33100 Udine, Italy
}

Particle transfer in the wall region of turbulent boundary layers is dominated by coherent structures which i) control the turbulence regeneration cycle, ii) bring particles toward and away from the wall and iii) favour particle segregation in the viscous region giving rise to nonuniform particle distribution profiles which peak close to the wall.

In this work, we focus on the transfer mechanism of inertial particles and on the influence of gravity on transfer, segregation and deposition mechanisms. By tracking $O\left(10^{5}\right)$ particles in Direct Numerical Simulation (DNS) of a turbulent channel flow at $R e_{\tau}=150$, we find that particles reach the wall mainly by two different mechanisms: free-flight, stronger for larger particles, and diffusional deposition, more significant for smaller particles. For dominant diffusional deposition, particles are segregated very near the wall and are then slowly driven to the wall.

\section{Introduction}

Turbulent particle transfer mechanisms in proximity of a wall are characterized by complex interactions between time-dependent turbulence structures and dispersed phase. Particles tend to accumulate in the viscous sublayer, where they remain trapped for long residence time. In particular, sweeps and ejections, which are instantaneous realizations of the Reynolds stresses, play a fundamental role in determining particle deposition rates $[4,9]$.

Particle transfer fluxes toward and away from the wall are dominated by coherent quasi-streamwise vortices [2,6,7], which play a fundamental role in the wall turbulence regeneration cycle $[3,5,12]$ and appear to have a triple effect: i) they cause the formation of particle streaks in the low-speed regions near the wall, ii) they create suitable conditions for particle entrainment and iii) they assist in deposition, by conveying particles from the outer flow to the wall region through sweeps, and eventually in particle resuspension, by conveying particles from the wall region to the outer flow through ejections.

\section{Numerical Simulation}

Particles are introduced is a Poiseuille turbulent plane channel flow of air $\left(\rho=1.3 \mathrm{~kg} / \mathrm{m}^{3}\right.$, $\nu=15.7 \cdot 10^{-6} \mathrm{~m}^{2} / \mathrm{s}$ ), assumed to be incompressible and Newtonian. We will consider two different flow configurations: upward vertical channel flow, with gravity acting along the negative streamwise direction $(x)$, and horizontal channel flow, with gravity acting 
along the negative wall-normal direction $(z)$. Periodic boundary conditions are imposed in both streamwise and spanwise $(y)$ directions and no-slip boundary conditions are enforced at the walls. Particle number density and particle size are assumed both small and there is no feedback of the particles onto the gas flow (one-way coupling).

The flow field was calculated by integrating the following non-dimensional mass and momentum balance equations (obtained using the duct half-width, $h$, and the shear velocity, $u_{\tau}=\sqrt{\tau_{w} / \rho}, \tau_{w}$ being the shear at the wall and $\rho$ the fluid density):

$$
\frac{\partial u_{i}}{\partial x_{i}}=0 ; \quad \frac{\partial u_{i}}{\partial t}=S_{i}+\frac{1}{R e_{\tau}} \frac{\partial^{2} u_{i}}{\partial x_{j} \partial x_{j}}-\frac{\partial p}{\partial x_{i}}
$$

where $u_{i}$ is the $i^{t h}$ component of the dimensionless velocity vector, $p$ is the fluctuating kinematic pressure, $S_{i}$ includes the convective term and the mean pressure gradient that drives the flow, and $R e_{\tau}=h u_{\tau} / \nu$ is the shear Reynolds number. Mass and momentum balance equations were solved directly using a pseudo-spectral method, based on transforming the field variables into wavenumber space, using Fourier representations for the $x$ and $y$ directions and a Chebyshev representation for the non-homogeneous $z$ direction. A two-level explicit Adams-Bashforth scheme for the nonlinear terms $S_{i}$ and an implicit Crank-Nicolson method for the viscous terms were employed for time advancement [13]

The shear velocity is $u_{\tau}=11.775 \cdot 10^{-2} \mathrm{~m} / \mathrm{s}\left(R e_{\tau}=150\right)$. and the mean velocity is $U=1.65 \mathrm{~m} / \mathrm{s}(R e=h U / \nu \simeq 2110)$. The computational domain was $1885 \times 942 \times 300$ wall units in $x, y$ and $z$ with $64 \times 64 \times 65$ nodes. The first collocation point away from the wall is at $z^{+}=0.18$. The time step used was $\Delta t^{+}=0.35325$ in wall time units.

Particles are injected into the flow at concentration low enough for particle-particle interaction to be negligible - dilute system conditions - and particles are assumed to be pointwise, rigid, spherical and to obey the following Lagrangian equation of motion:

$$
\frac{d \mathbf{v}}{d t}=\frac{C_{d}}{\tau_{P}}(\mathbf{u}-\mathbf{v})+\left(1-\frac{\rho}{\rho_{P}}\right) \mathbf{g}-\xi(\epsilon) \frac{6.46}{12 \pi} \frac{d_{P}}{\tau_{P}}\left|\frac{\partial u_{x}}{\partial z}\right|^{\frac{1}{2}}\left(v_{x}-u_{x}\right) \mathbf{e}_{\mathbf{z}}
$$

in which $\mathbf{v}$ is particle velocity vector, $\mathbf{u}$ is fluid velocity vector at particle location, $C_{d}=24\left(1+0.15 R e_{P}^{0.687}\right) / R e_{P}$ is the Stokes drag coefficient, $\tau_{P}=d_{P}^{2} \rho_{P} / 18 \rho$ is the particle relaxation time ( $d_{P}$ and $\rho_{P}$ being particle diameter and density respectively), $\mathbf{g}$ is gravitational acceleration and $\mathbf{e}_{\mathbf{z}}$ is the unit vector in wall-normal direction. All physical quantities have been made dimensionless in terms of wall units based on $u_{\tau}, \rho$ and $\nu$.

The LHS of Eq. 2 represents particle inertia, and the terms on the RHS of Eq. 2 represent the effects of Stokes drag, gravity and Saffman lift force. Other forces acting on the particle are assumed to be negligible [1].

In the present simulations, $48^{3}$ flyash particles (with $\rho_{p} / \rho=769.23$ ) have been released randomly within the computational box. The diameters of the tracked particles are $d_{p}=220,110,40,30,20$ and $5 \mu \mathrm{m}$, corresponding to the following non-dimensional particle relaxation times: $\tau_{p}^{+}=116.3,29.1,3.8,2.0,0.89$ and 0.0556. Particle trajectories were tracked through integration of Eq. 2 by an explicit method, using the channel flow DNS code to supply the fluid velocity field at each time step. The initial velocities of the particles were set equal to the interpolated fluid velocities at each particle location. Eq. 2 does not include wall effects: a particle is elastically reflected when its centre is less than a distance $d_{P} / 2$ from the wall. Fluid forces acting on particles are calculated with a Lagrange interpolation of order three. 


\section{Results and Discussion}

We report here some of the results presented in [8], relative to turbulent transfer mechanisms and segregation of inertial particles in a boundary layer of a vertical upward channel flow. Particles tend to accumulate in a near-wall region, where the particle number density profile develops a maximum, which shifts toward the wall over time $[8,9,11]$. This behavior is due to non-uniform turbulence advection mechanisms, whose intensity decreases to very low values in the near wall region. The mechanisms leading to particle wall-ward migration in turbulent boundary layer (turbophoresis [11]) are investigated by examining particle dynamics in connection with the dynamics of wall coherent structures.

\subsection{Turbulent Structures and Particle Trapping Mechanism}

A strong correlation exists between particle fluxes to the wall and high wall shear stress regions, corresponding to sweep events; low wall shear stress regions, corresponding to ejections, correlate well with off-the-wall particle fluxes. Since particle distribution in the wall normal direction increases in the wall layer and decreases in the outer layer, fewer particles will be available to be transferred to the wall. The efficiency of sweep/ejection events is thus conditioned by the presence of particles. Also, fluxes to the wall always have a greater intensity compared with fluxes toward the outer flow: particles tend to settle in a sediment layer at the wall, which roughly corresponds to the viscous sublayer. For upflow configuration, gravity can not directly cause particle deposition at the wall and the build-up of particles in a sediment layer must be explained by different mechanisms.

The structures which dominate the wall layer dynamics are quasi-streamwise vortices $[8,12]$, which generate sweeps on the downwash side and ejections on the upwash side. In turn, ejections contribute to the maintenance of the lifted low-speed streaks.

We examined a large number of snapshots showing the action of quasi-streamwise vortices on particle transfer in the wall region. We show one of these in Figure 1, which focuses on a $y-z$ window of the computational domain extruded for the length of one streamwise cell at time $t^{+}=1412$. The main character in this picture is the green counterclockwise-rotating quasi-streamwise vortex, centred at $z^{+}=36$ : its action in transferring the black particles (having negative $w_{P}^{+}$) to the wall and the blue particles (having positive $w_{P}^{+}$) away from the wall is apparent.

A secondary, but relevant, character in this picture appears as a red isosurface identifying a smaller counter-rotating quasi-streamwise vortex, centred at $z^{+}=9$ and extending well into the viscous wall layer [3]. As is apparent from Figure 1, the role of the secondary newly-born vortex is crucial in preventing particles from being entrained in the outer flow. The combined action of the newly-born vortex and the mature vortex prevents particles from being entrained in the outer flow by reducing the width of the 'ejection avenue' through which particles in the wall layer have to pass to reach the outer flow.

Particles with $\left|w_{P}\right|<10^{-3}$ (empty circles) are mostly settled under the low-speed streak (blue isosurface) in a wall layer confined between the newly-born vortex and the wall. The situation just described has statistical relevance [8].A sample analysis conducted for larger inertia particles $\left(\tau_{p}^{+}=29.1\right.$ and $\left.\tau_{p}^{+}=116.3\right)$ showed that particles segregation mechanisms are similar for the particle time-scales investigated.

In the light of this scenario, a particle residence time analysis was conducted to gain further insight on the effect of near-wall turbulence structures on depositing particles. Fo- 


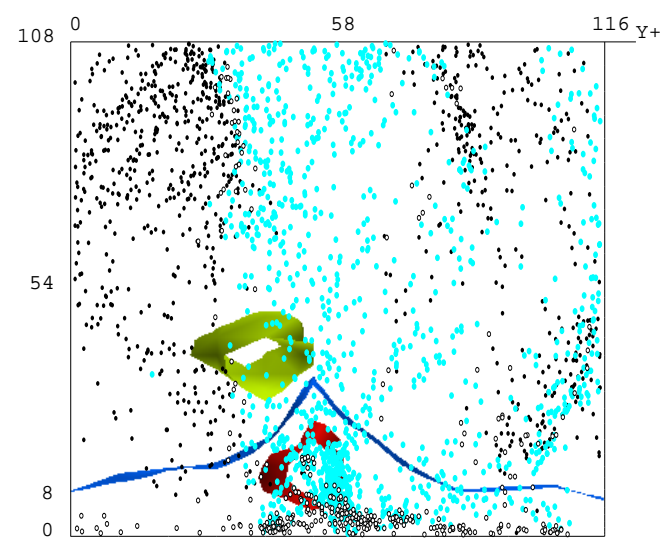

Figure 1: Particle distribution and turbulent coherent structures in near-wall region.

cusing on a thin slab of fluid close to the wall, 3 wall units thick, we followed each particle path to the wall and we recorded the time spent before deposition. If a particle escapes the slab before depositing (due to re-entrainment), the time counter for that particle is reset to zero. We tried to find a relationship between the non-dimensional particle residence time $\left(T_{r e s}^{+}\right)$in the slab and particle wall-normal velocity at deposition $\left(w_{d e p}^{+}\right)$.

In Figure 2, we show a scatter plot of $T_{r e s}^{+}$versus $w_{d e p}^{+}$for the three particle sets. We can identify two distinct populations: free-flight particles, whose velocity is larger than the near-wall fluid velocity fluctuations, and diffusional deposition particles, whose velocity is roughly equal to the near-wall fluid velocity fluctuations [10].

Focusing on the monitor slab and considering only the motion in the wall normal direction, the free-flight deposition mechanism can be predicted by the ballistic equation $d w_{p}^{+} / d t=-w_{p}^{+} / \tau_{p}^{+}$. Solving for a particle controlled by the Stokes drag, entering the monitor slab with wall-normal velocity $w_{p}^{+}\left(z^{+}=3\right)$ at time $t^{+}=0$ and depositing with wall-normal velocity $w_{d e p}^{+}=w_{p}^{+}\left(z^{+}=d_{p}^{+} / 2\right)$ at time $T_{r e s}^{+}$, we find:

$$
\tau_{p}^{+} w_{d e p}^{+}\left[1-\exp \left(\frac{T_{r e s}^{+}}{\tau_{p}^{+}}\right)\right]=3-d_{p}^{+} / 2
$$

where $3-d_{p}^{+} / 2$ on the right-hand side is the actual slab height chosen for the residence time analysis [10].We plotted Eq. 3 in Figure 2. Apparently, Eq. 3 predicts well the behavior of particle depositing with large velocity and largerly underpredicts deposition times for particles depositing by diffusion mechanism. There is evidence of an effect of particle relaxation time: small particles (Figure 2a) deposit almost exclusively by diffusion, whereas for large particles (Figures $2 \mathrm{~b}$ and $2 \mathrm{c}$ ) free-flight deposition is dominant.

\subsection{Effect of Gravity on Deposition}

To analyze the effect of gravity on particle transfer and segregation, we compared particle behavior in both upward and horizontal channel flow configurations. The most interesting results where obtained using particle samples with small inertia.

In Figure 3, we show the time evolution of concentration profiles for $\tau_{p}^{+}=0.0556$, $\tau_{p}^{+}=0.89$ and $\tau_{p}^{+}=2.0$ particles. Starting from the initial uniform distribution, concentration profiles were computed at fixed time intervals by subdividing the channel into 

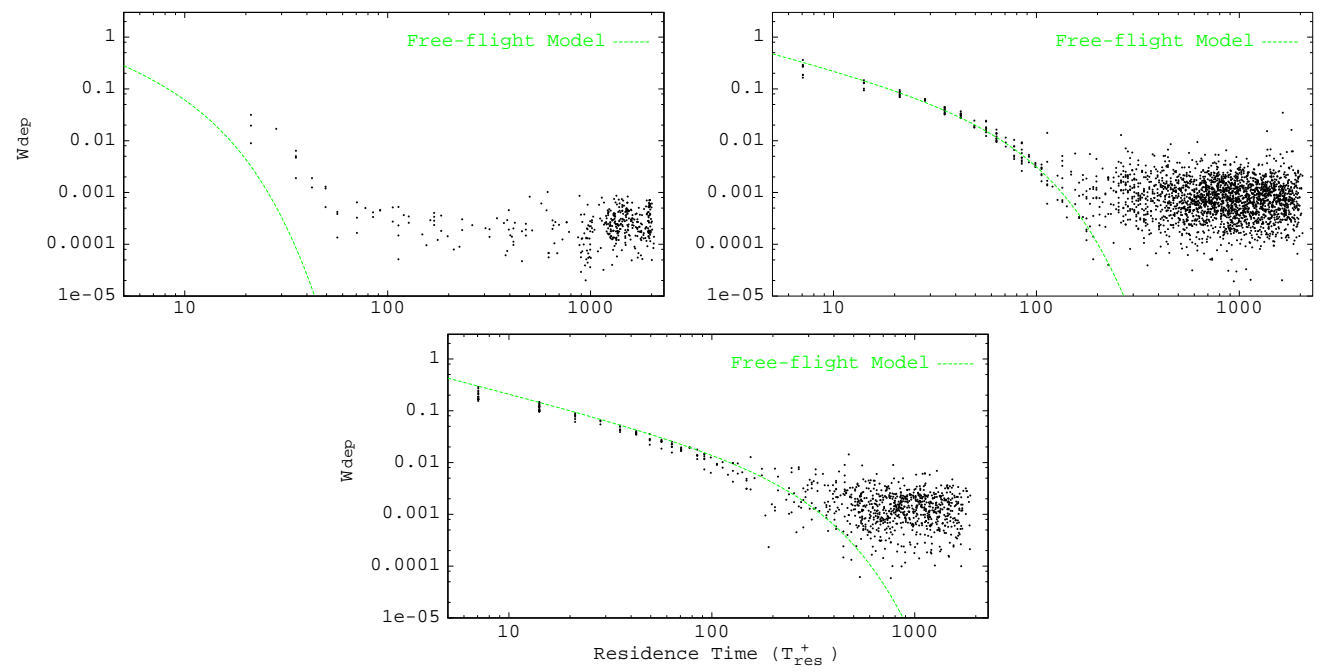

Figure 2: Residence time of particles in a slab of $z^{+}<3$ versus wall-normal deposition velocity. a) $\tau_{p}^{+}=3.8$ particles, $\left.b\right) \tau_{p}^{+}=29.1$ particles, $\left.c\right) \tau_{p}^{+}=116.3$ particles.

65 slabs (through Chebyshev polynomials) and counting the fraction of particles that fell within each slab. Concentration profiles for upward vertical channel flow (Figures 3a, 3c and $3 e$ ) exhibit the expected accumulation in the near-wall region.

Figures $3 \mathrm{~b}, 3 \mathrm{~d}$ and $3 \mathrm{f}$ refer to particle number density distribution near the bottom wall of the horizontal channel. Due to viscous diffusion, the concentration profile for $\tau_{p}^{+}=0.0556$ particles develops a peak which increases in time and shifts toward the wall (Figure 3b). After an initial transient of about 600 wall time units, a second peak appears right at the wall, due to gravity force which acts to deposit large fractions of particles trapped in the viscous sublayer. A similar trend is observed for $\tau_{p}^{+}=0.89$ particles (Figure $3 \mathrm{~d}$ ) and for $\tau_{p}^{+}=2.0$ particles (Figure $3 \mathrm{f}$ ). However, the effect of gravity on larger inertia particles becomes significant at earlier stages of the simulation (roughly $\left.t^{+} \leq 100\right)$ and the peak at the wall takes up more quickly. If we further increase particle inertia, the diffusion-induced peak of concentration still exists but it is completely hidden by the gravity-induced one.

Notice that, after 1200 wall time units from the beginning of the simulation, the maximum intensity peak is obtained for $\tau_{p}^{+}=0.89$ particles.

\section{Conclusion}

We examined the mechanisms leading to transfer, segregation and deposition of inertial particles at the wall, focusing on the role of turbulence coherent structures.

First, we found that i) a strong correlation exists between sweep events and particle flux toward the wall, and between ejection events and particle flux toward the outer flow, ii) the efficiency of sweep/ejection cycle decreases for increasing particle inertia, i.e. for increasing ratio of particle to fluid time-scale [8].

Second, we emphasized the relevance of a smaller streamwise oriented vortex in reducing the width of the 'ejection channel' so that only particles entering the wall-layer 


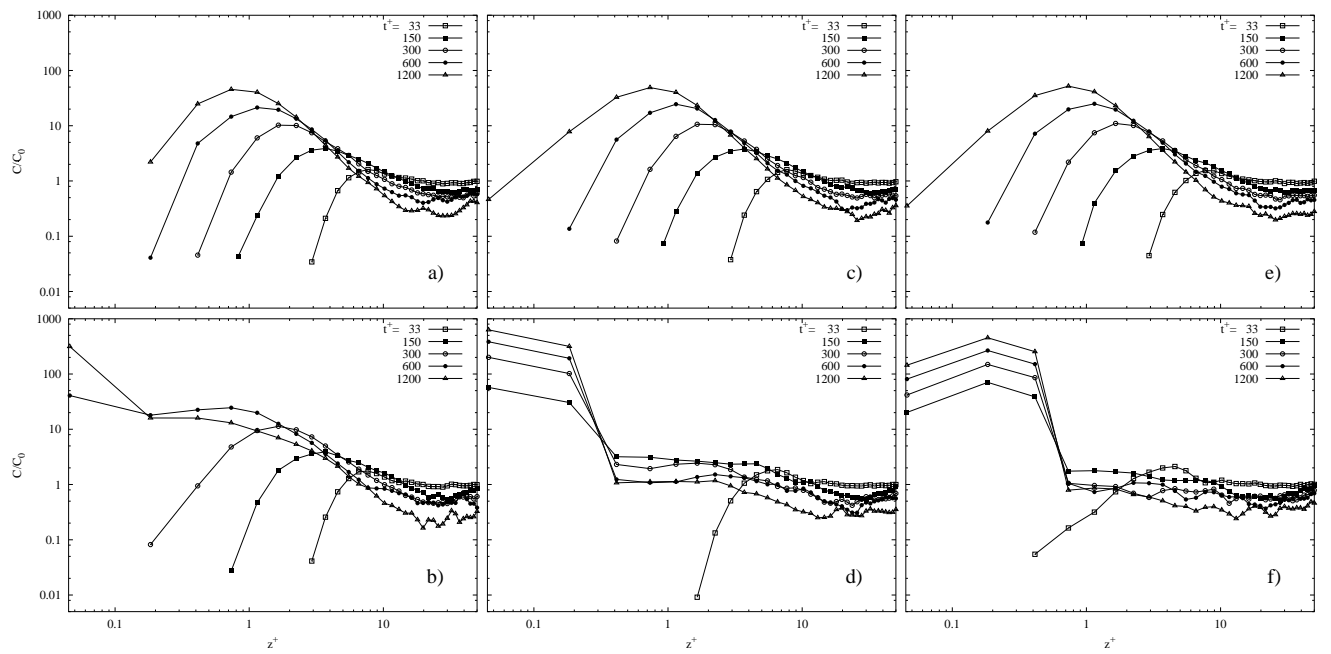

Figure 3: Time evolution of mean concentration profiles: a) vertical channel, $b$ ) horizontal channel (bottom wall). a), b) $\left.\left.\left.\left.\tau_{p}^{+}=0.0556 ; c\right), d\right) \tau_{p}^{+}=0.89 ; e\right), f\right) \tau_{p}^{+}=2.0$.

with specific trajectory curvature may be re-entrained into the outer flow.

Further analysis revealed a clearcut distinction between particles depositing by freeflight mechanism (which can be described through a simple one-dimensional ballistic equation) and particles depositing by diffusional deposition mechanism (due to the small fluctuations in the viscous wall region). The balance between these two mechanisms depends on particle inertia. Deposition of small inertia particles (namely $\tau_{p}^{+} \leq 5$ ) is dominated by diffusion phenomena, free-flight deposition mechanism becomes important for increasing partile inertia.

Finally, we analyzed the effect of gravity on particles depositing by diffusional deposition. Results on mean particle concentration along the wall-normal direction reveal the existence of a two-stage process in which viscous diffusion creates a first peak of particle concentration in the near-wall region and gravity force creates a second peak at the wall.

\section{References}

[1] Armenio, V., and Fiorotto, V., 2001. Phys. Fluids A, 13, 2437.

[2] Brooke, J.W., Kontomaris, K., Hanratty, T.J., and McLaughlin J.B., 1992. Phys. Fluids, 4, 825.

[3] Brooke, J.W., and Hanratty, T.J., 1993. Phys. Fluids A, 5, 1011.

[4] Cleaver, J.W., and Yates, B., 1975. Chem. Eng. Science, 30, 983.

[5] Jimenez, J., and Pinelli, A., 1999. J. Fluid Mech., 389, 335.

[6] Kaftori, D., Hetsroni, G., and Banerjee, S., 1995a. Phys. Fluids, 7, 1095.

[7] Kaftori, D., Hetsroni, G., and Banerjee, S., 1995b. Phys. Fluids, 7, 1107.

[8] Marchioli, C., and Soldati, A., 2002. J. Fluid Mech., 468, 283.

[9] McLaughlin, J.B., 1989. Phys. Fluids, 1, 1211.

[10] Narayanan, C., Lakehal, D., Botto, L., and Soldati, A., 2003. Phys. Fluids, 15, 763.

[11] Reeks, M.W., 1983. J. Aerosol Sci., 14, 729.

[12] Schoppa, W., and Hussain, F., 1997. In Self-sustaining Mechanisms of Wall Turbulence (ed. R. Panton), Advances in Fluid Mechanics, vol. 15, 385.

[13] Soldati, A., and Banerjee, S., 1998. Phys. Fluids, 10, 1742. 